\title{
Designing An Electronic Shoe To Measure Some Mechanical Values Of Movements Of The Legs, Supported By Wireless Transmission "Dr / Osama El-sayed Tmmam
}

\section{Introduction:}

Engineering applications have been increased widely in sports field in the recent years. Most of the researches now days depend some how on the electronics devices. These devices could provide an accurate information with fast response to even small change and events. Which could record, analysis and make decisions in some cases. With the help of these powerful and mostly small tools the sports have been enhancement. A force plat form is one of devices that have been use for gait analysis to measure the force applied on it [1-4]. Moreover, EMG is used to analysis the electric signal of the human muscles which helped to understand the ability of the human muscles to do works and recognize the normal and damaged muscles [5-8]. On top of that, the foot scan devices provide pressure distribution under the legs and used for balancing motion and dynamics analysis. In the quart, it also has been used for medical application such as recognizing the diabetic foot [9-12].

The using of electronics devices is not only considered for analysis task. The judgment and accurate decision are improved with the help of the engineering application such as image processing which is used in football as VAR since 2017/2018, in javelin to detect the correct throw and measure the distance of the thrown spear and many other sports[13-16]. The direction now days is to force the electronics device to be an essential part in all sport fields to analysis, judge and even forecast the performance of the human and find the optimal and best performance.

\section{The search goal}

- Lecturer of Biomechanics- Wrestling in Department of Sports Training and Movement Science Faculty of Physical Education Assiut University 
The search is focusing on the force measurements in the two measurements direction $\mathrm{x}$ and $\mathrm{y}$. It's provide a fast way to record every event with high accuracy

\section{The search questions}

Is it able to measure some mechanical variables ?

Could we use wireless communication system and build a database for the old cases?

\section{Search subject}

The search used the experimental way to prove the search results and ability to investigate

\section{Search community}

The search focus on the professional players in the Egyptian national team of Roman Westlen sport

\section{Search test population}

The test trainer choose such that different weight are used and different categories The paper sections are, section I describe the main four units and the design of each unit's section II calibration of each unit, section III result, section IV references

\section{1- Electronic shoes design:}

In this section a description of the main component and the design steps are provided. The system comprises of 4 main devices. Two of them act as sensor devices which responsible about collecting data from the surrounded field. These data are the force, angles and acceleration. One other unit is a communication unit which is responsible about measuring sending data to the fourth unit. The fourth unit is the Micro controller which responsible about the most important tasks analysis and sending to the mobile phone the information.

\section{A. Force measurement unit:}

The FSR is a force sensor that use a variable resistance which change with changing the applied force on a specific area. Customized design is available and easy to make. The sensor two face are contact with two electrode surfaces with neglected resistance of very low value. However, due to its easy it used in widely application.

For the experiment purpose, two electrodes are designed to measure the average applied force on the sensor area. An insole with size of 41 is used as a sensor with dimension of $23 * 9.5 \mathrm{~cm} * \mathrm{~cm}$ as shown in fig (1) for the force calculation. The two electrodes 
are design with the same dimension and a flexible PCB are made for that task. A protection layer of booth cupper and flexible plastic are inserted above the two electrodes to increase the lifetime and provide more mechanical stress resistance during the operation on the electronics shoes. A polymer of non-linear force- resistance characteristic is used which is essentially a carbonic bond crystal structure with 4 covalent bonds with adjacent atoms. Velostat is very good choice even with the high cost consider compared with other material but due to the chemical, stress and strain stability of velostat, it is providing a good choice for the material to be use in dynamic application.

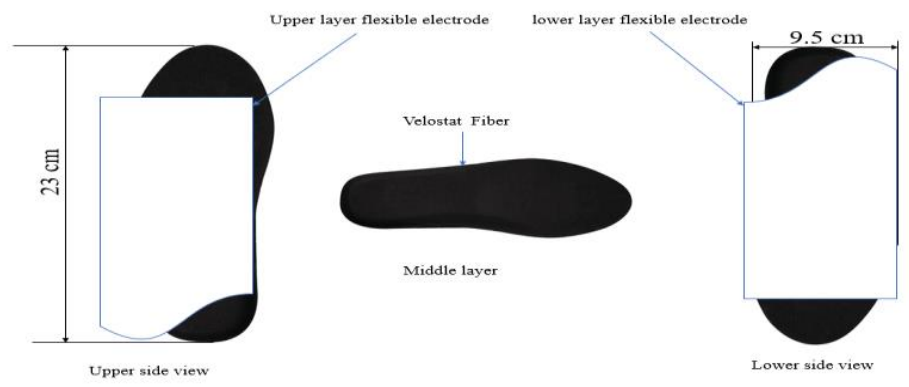

Fig (1)

The characteristic of the FSR sensor are mostly not linear with the resistance and linear with conductance. Most research use the nonlinear model of the resistance as standard [17]. The basic equation form is presented below:

$$
F=\left(\frac{A}{R}\right)^{\frac{1}{B}}
$$

This equation can be shown by the fig (2). As it is shown in the curve the value of the resistance is very high at low force applied and decrease rapidly with increase the applied force. Three important point 


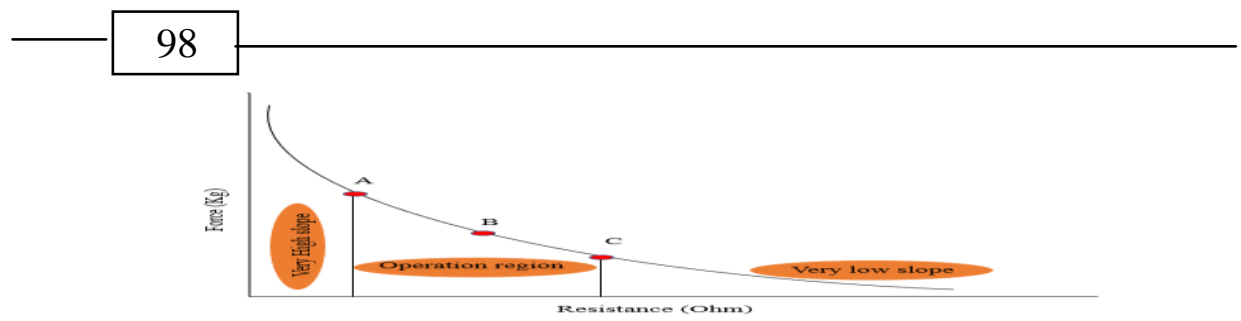

Fig (2)

$\mathrm{A}, \mathrm{B}$ and $\mathrm{C}$ are shown in the curve. The slope before the point A has a very large value. That is mean when only small amount of force is applied the resistance change with very large value and for this reason the region before point $\mathrm{A}$ cannot be use as operation region. In the other hand, the slope after the point $\mathrm{C}$ approximately equal to zero and due to this very large change in the applied force will cause only small change in the resistance value and a very high resolution measuring analog to digital converter will needed which will increase the product cost very much. Due to

(2)

By using the conductance instated of the resistance, the force-conductance relation is lineariz

ed, and this, this region also cannot be use. The active or effective operation region is the region between the point $\mathrm{A}$ and $\mathrm{C}$ and the resistance value at point $B$ is mostly taken for the analog to digital converter voltage divider (ADC).

One way to overcome the non-linearity problem of the used material is to use the conductance instated on the resistance. Since the relation between the resistance and the conductance can be described by:

$$
G=(R)^{-1}
$$

the operation range increased. The relation is shown by fig (3)

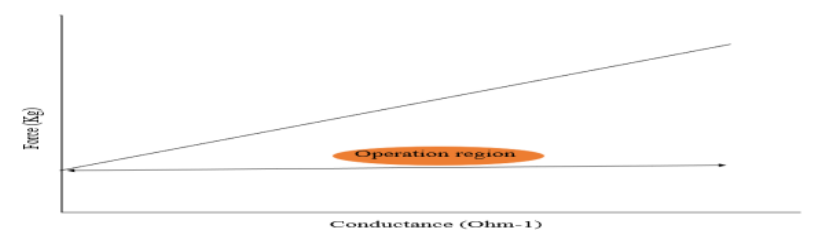

Fig (3)

Assiut Journal For Sport Science Arts 


\section{B- Inertial measurement unit (IMU):}

The inertia measurement unit is a type of sensor is a sensor package containing 3 discrete sensors that can be used to track movement and orientation of objects by measuring acceleration and angles in different movement direction. The acceleration is measured by $\mathrm{m} / \mathrm{s}^{\wedge} 2$ or $\mathrm{g}$ units where both are equivalent. Fig (4) shows an example of IMU sensor which cost around 140
$\$$. The selection of IMU sensor can be summarized in 5 main factors price, range of operation, resolution, degree of freedom and working signal (analog or digital). Moreover, another consideration is taken in account such as noise disturbance, power absorbed and operation temperature range.

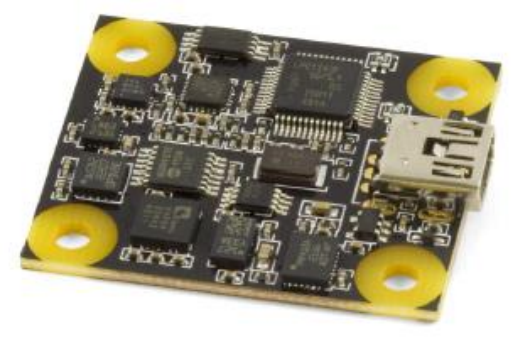

Fig (4)

One IMU is used for each leg. The following part will explain how the IMU sensor is used to measure both angle and acceleration in specified direction.

(3)

Where $\omega^{I}$ is the actual measured angular $\omega$ is the true value, $\beta$ is the bias and $\gamma$ is white noise error represented by zero mean gaussian function. By using the general
Angle measurement:

Angular moment which provided from the sensor can be described by eq (3):

$$
\omega^{I}=\omega+\beta+\gamma
$$

integration of the angular m0omentum the angle at certain time $t$ can be approximately be describe by eq (4): 
$\theta(t+\Delta t)=$

$\theta(t)+\frac{d}{d t} \theta \Delta t+E$

\section{(4)}

$\frac{d}{d t} \theta=\omega$

With tacking initial set point as reference, the angle can be started to calculate from this point and at any time $\mathrm{t}$ the angles can be calculated. The error in this case will be accumulated and will increase Acceleration measurement:

The acceleration provided by the sensor is following by the eq(6):

$$
a^{I}=a g+a l+n
$$

Where $a^{T}$ is the actual measured acceleration $a g$ is the gravity acceleration which equal $9.81 \mathrm{~m} / \mathrm{s}^{\wedge} 2, \quad n$ is the white noise error represented by zero mean gaussian

function. Using analysis for the $3 \mathrm{D}$ system the angle at each direction can be defined with the information of acceleration as:

$$
\theta z=-\tan ^{-1}\left(\frac{a_{x}}{a_{y}}\right)
$$

(8)

$$
\theta x=-\tan ^{-1}\left(\frac{a_{z}}{\left(\left(a_{x}\right)^{2}+\left(a_{y}\right)^{2}\right)^{0.5}}\right)
$$

By using eq (7) and (8) the angle at each direction can be measure.

\section{Bluetooth module:}

Normal Bluetooth module is used for profuse of sending data from the Micro controller to the mobile phone. A
Bluetooth module $\mathrm{Hc}-05$ is used due to the low cost and high reporting rate. Fig (5) shows the module. 


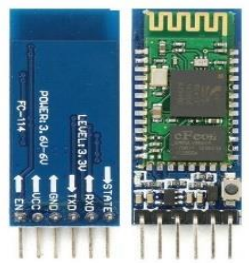

Fig (5)

The reporting rate is adjusted by programing the microcontroller. During the test its shows that 50 sample per second is the maximum rate that could be send in each second. This behavior back to two reasons, first is the time required of analysis for controller to read, analysis and communicate correctly and the second reason depend on the available storage system in the receiving phone. More sending time means more storage data and lack in reporting.

\section{B- Micro controller (Arduino mega 2560):}

Micro controller is the brain of any electronic device which use to store, analysis and send communication between the different module of the system. Due to the large data and analysis process Arduino mega 2560 is used for this task while Arduino uno storage memory couldn't did the needed task. Fig (6) shows the Microcontroller which used in this paper.

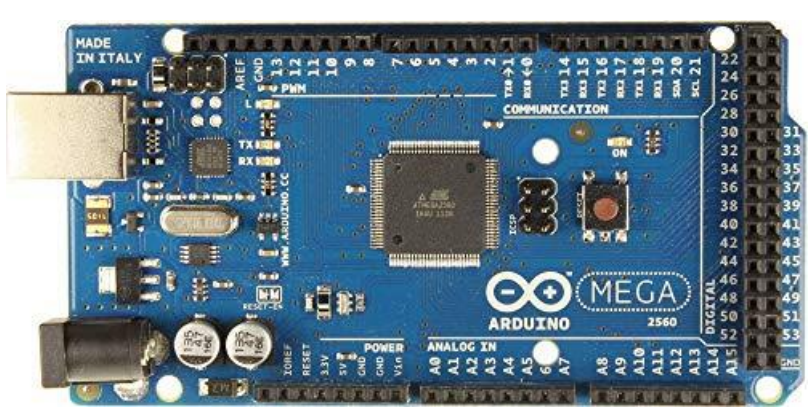

\section{Fig (6)}

Reading the analog signals from both FSR sensor and convert the signal to digital for more analysis is the first task. The kit alloys high speed recording with sampling frequency could reach to 14 GHZ. Second task of the controller is to measure the signal from both IMU and 


\section{2}

establish calculating angles and acceleration by the equation describe in the upper section. The data are then sent by the Bluetooth to mobile android application named Bluetooth terminal $\mathrm{HC}-05$ can be download by play store and work at any android phone and IOS phone. This make the device able to work for any phone. Below section provide the signal recording method and connection diagram.

\section{* FSR Signal recording.}

In purpose to record the data and analysis the signal from the FSR with the Arduino mega, A voltage divider technique with $220 \mathrm{Ohm}$ resistance are used

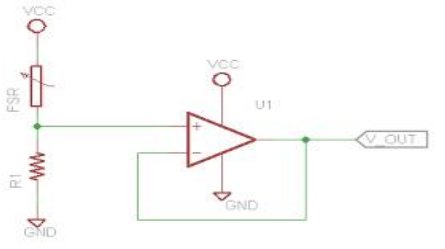

for analog signal read. The Analog signal are scaled to $1023=2$ power 10 due to the 10 bit microcontroller. $\mathrm{Vcc}=5$ volt is applied and producing a resolution of $5 / 1024=4.8 \mathrm{mV}$ between each level. The next figure shows the voltage divider circuit techniques using the operational amplifier to prevent any leakage current.

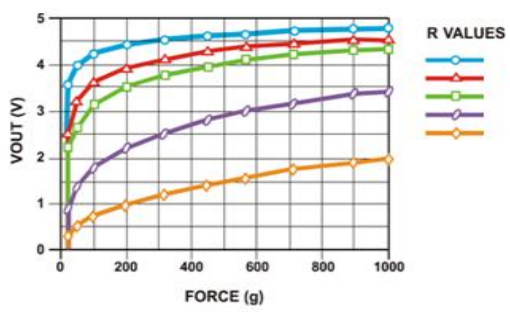

Fig (7)

Fig (8)

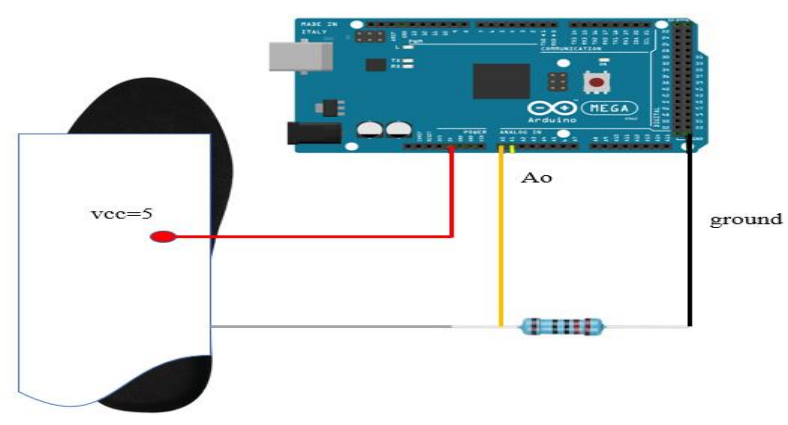

Assiut Journal For Sport Science Arts 
The signal is the scaled to 5-volt scale by dividing the analog value by 1023 and multiply by 5 to present for 5 scale voltage. The choice of 220-ohm resistance is based on

(9)

By using eq (9) FSR resistance can be calculated. With accurate calibration the relation * IMU Signal connection:

Fig (9) represent the connection diagram of the IMU with the Arduino mega.

\section{Calibration:}

$$
F S R=220 *\left(\frac{V c c}{V o u t}-1\right)
$$

microcontroller limitation current not accessed $10 \mathrm{~mA}$ at full loading. The FSR value resistance can be calculate using the equation written down

between the FSR resistance and the applied force on the FSR can be driven.

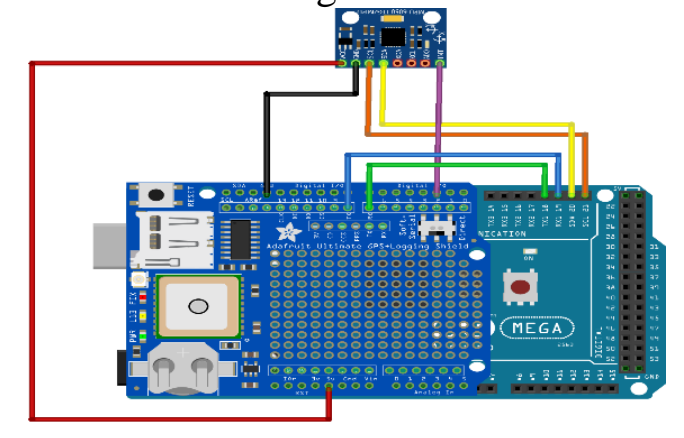

Fig (9)

\section{a- FSR}

The calibration is made shown for the both legs in the with standard weight start from $6 \mathrm{Kg}$ to the value of $80 \mathrm{Kg}$ and the conductance force characteristics are plotted as fig 10 and 11 . Table 1 represented the calculated value of the test calibration 


\begin{tabular}{|c|c|c|}
\hline 104 & Table (1) & \\
\hline \multicolumn{3}{|c|}{ Force- conductance calibration test characteristics } \\
\hline Force $(\mathrm{kg})$ & $\begin{array}{l}\text { Conductance (mho) } \\
\text { right leg sensor }\end{array}$ & $\begin{array}{c}\text { Conductance (mho) } \\
\text { left leg sensor }\end{array}$ \\
\hline 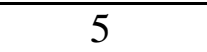 & 0.034955 & 0.006843 \\
\hline 10 & 0.047031 & 0.010349 \\
\hline 15 & 0.070342 & 0.01582 \\
\hline 20 & 0.092593 & 0.018393 \\
\hline 25 & 0.113855 & 0.021581 \\
\hline 30 & 0.128472 & 0.0267 \\
\hline 35 & 0.154784 & 0.028488 \\
\hline 40 & 0.172325 & 0.03239 \\
\hline 45 & 0.194252 & 0.035419 \\
\hline 50 & 0.207407 & 0.044039 \\
\hline 55 & 0.222443 & 0.045489 \\
\hline 60 & 0.239791 & 0.04784 \\
\hline 65 & 0.260031 & 0.056487 \\
\hline 70 & 0.283951 & 0.057633 \\
\hline 75 & 0.312654 & 0.058824 \\
\hline 80 & 0.347737 & 0.06135 \\
\hline 85 & 0.39159 & 0.064092 \\
\hline
\end{tabular}

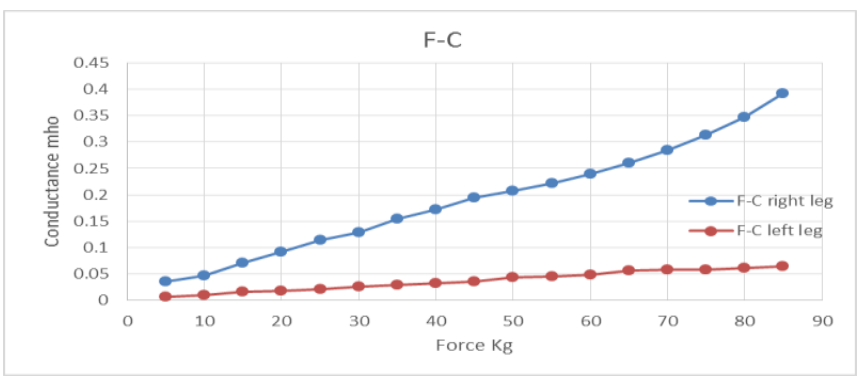

Fig (10)

Using least square technique to estimate each sensor characteristics are the solution to present the sensor characteristics. The linear characteristics curve shown by dot line in fig (11) 


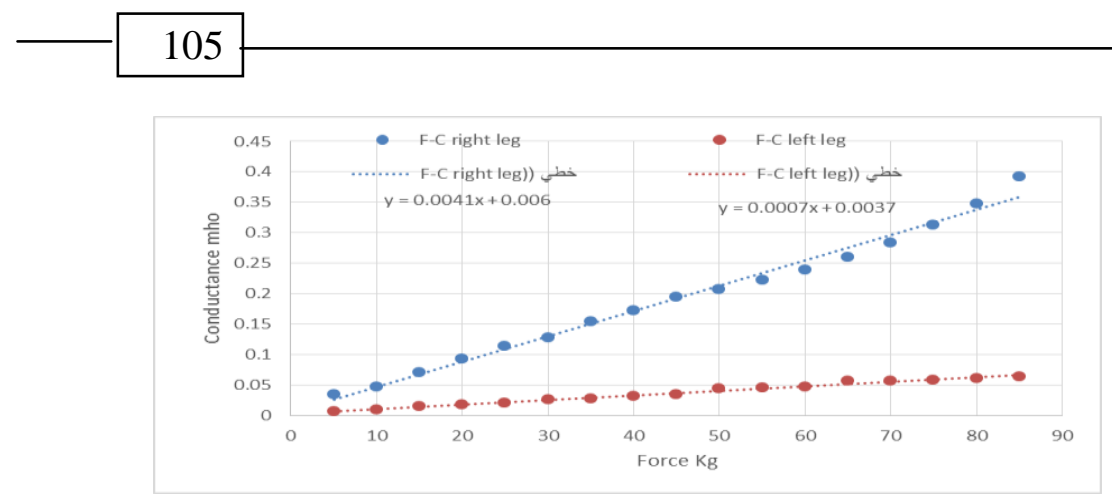

Right leg sensor

$$
\begin{gathered}
\text { Fig (11) } \\
F=243.902439 * G-1.4634
\end{gathered}
$$

\section{Left leg sensor}

Both sensors show stable and low error until $55 \mathrm{KG}$ applied force after $80 \mathrm{Kg}$ the error increase rapidly and nonaccurate result will be for the right leg sensor while left leg sensor still stable and with accepted error in full region. Due to this, maximum operation range is desired to be less than $64 \mathrm{Kg}$ for low error and accurate measurement. for the full-scale measurement range the RMS Error is $4,1 \%$ for right leg sensor and 3\% for left leg sensor inf the nonlinear characteristics is used. But in case of replace the resistance with the conductance the error is reduced to 3.28157 $\%$ for the right leg and $2.5 \%$ for the left leg. that range value the resistance value approximately is (20-110) to
(10)

(2-13) ohm for Right and left leg sensor.

\section{b- IMU:}

The calibration of the IMU is only about how to design the PID controller of the sensor to minimize the error as possible and faster response is reached. The steps can be summarized in the following: 1-Change the gain of the $\mathrm{KI}$ until the system is oscillated.

2-change the gain of the $\mathrm{Kd}$ until the noise is reduced.

3 -select a value in between these two gains using try unit stifled measurement are achieved

\section{Result:}

The result will be summarized as steps that made after the calibration to test the electronics shoes. 
1. The code is uploaded to the controller so it can start the main function.
2. Android program for Bluetooth communication is downloaded from play store shown in fig (12)

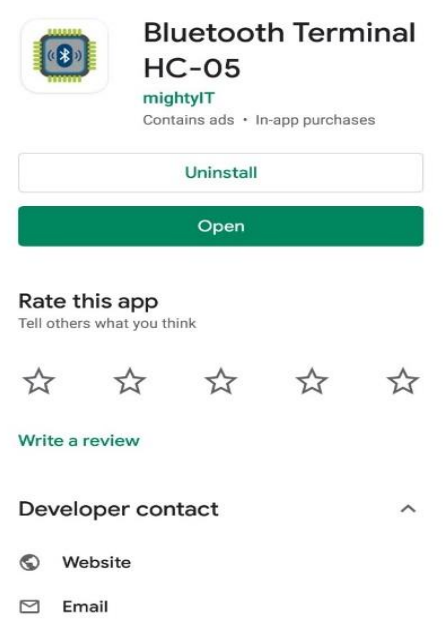

\section{Fig (12)}

3. After download the HC-05 signal for communicate program, scan for the available as shown down in fig (13). devices and select the

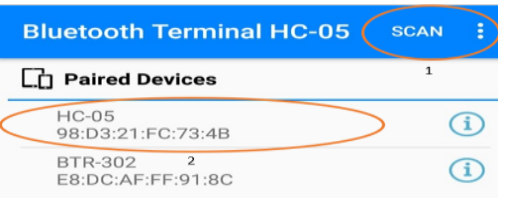

Fig (13)

4. Cheek the connected the sensor position must not signal and the system will need around 1-2 second to establish the calculation during this time change. Fig (14) shows the step 4 and data sending 


\section{7}

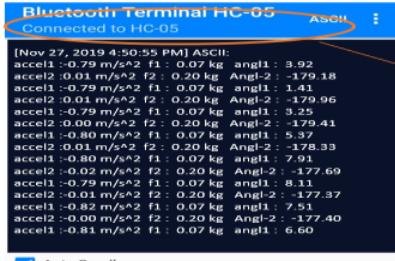

Auto Scroll
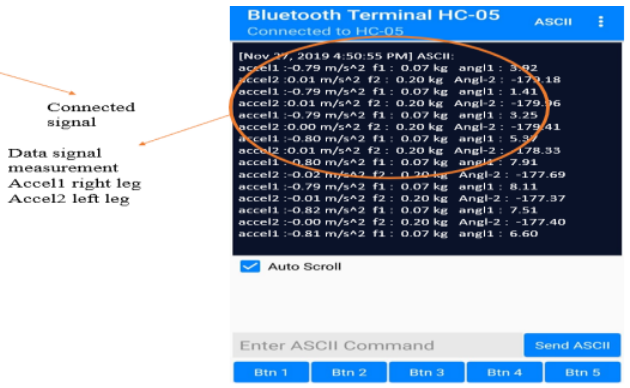

Fig (14)

5. The data storage can be send using send option as shown in fig (15)

\section{Fig (15)}

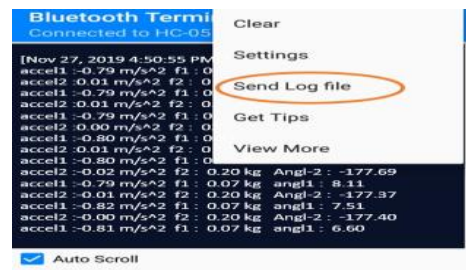

\section{IMU angle measurement test in fig (16)}
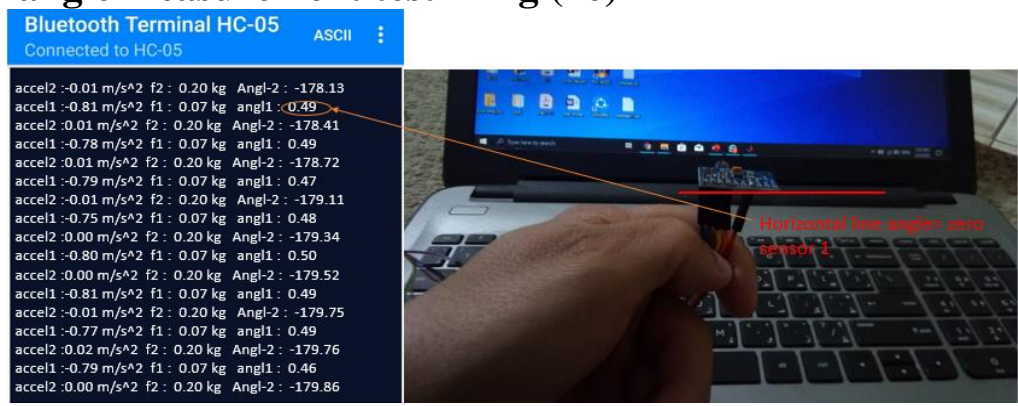

Fig (16) a (angle = zero)

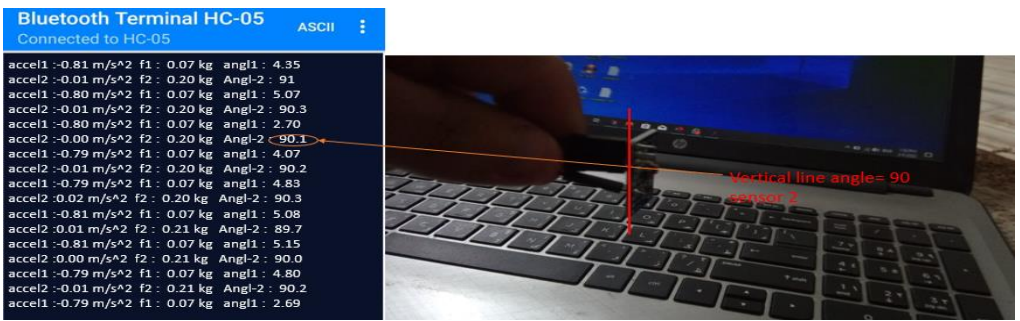

Fig (16) b (angle = 90)

Assiut Journal For Sport Science Arts 


\section{8}
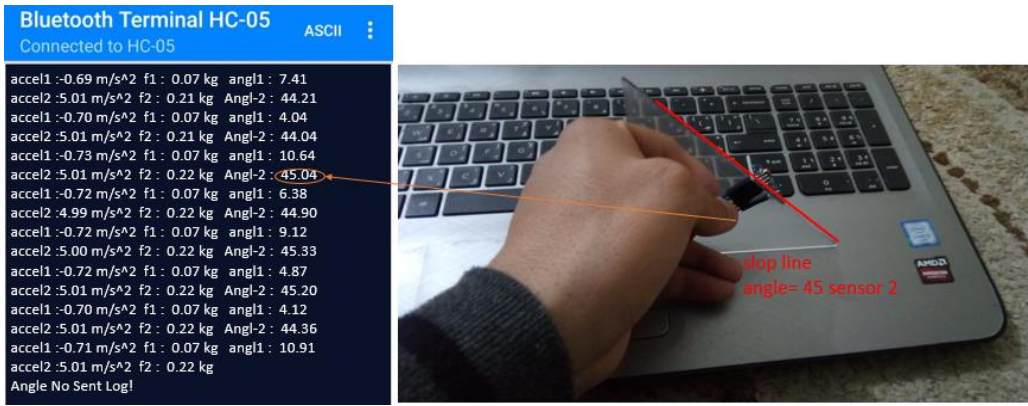

Fig (16) b (angle = 45)

\section{Case study using the electronics device:}

In this part an experiment is made. By let a trainer of $75 \mathrm{~kg}$ walk with the device and record the data. After receiving the data its analyzed and information are extract and present below. Fig (17) and 18 shows 3 main frames of recorded video for the analysis. The first frame shows the right leg start to touch the ground with angle of
22 degree. While the second frame shows the right angle with full stand on ground level and angle equal to zero with maximum weight stand on right leg equal to $40 \mathrm{~kg}$. At this moment the left leg start leaves the ground with negative angle. The left leg is then stand on the ground with total force of 41 $\mathrm{Kg}$.
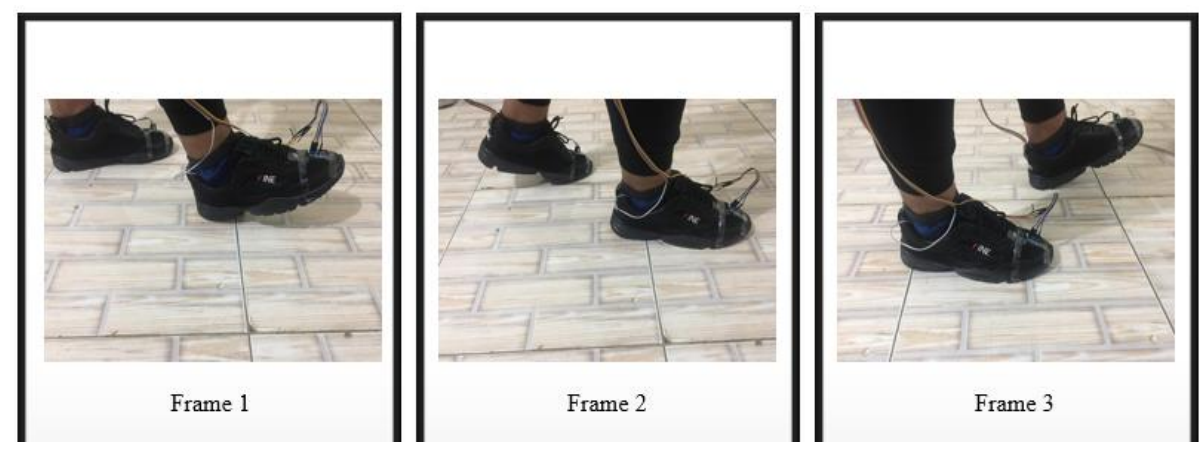

Fig (17) 


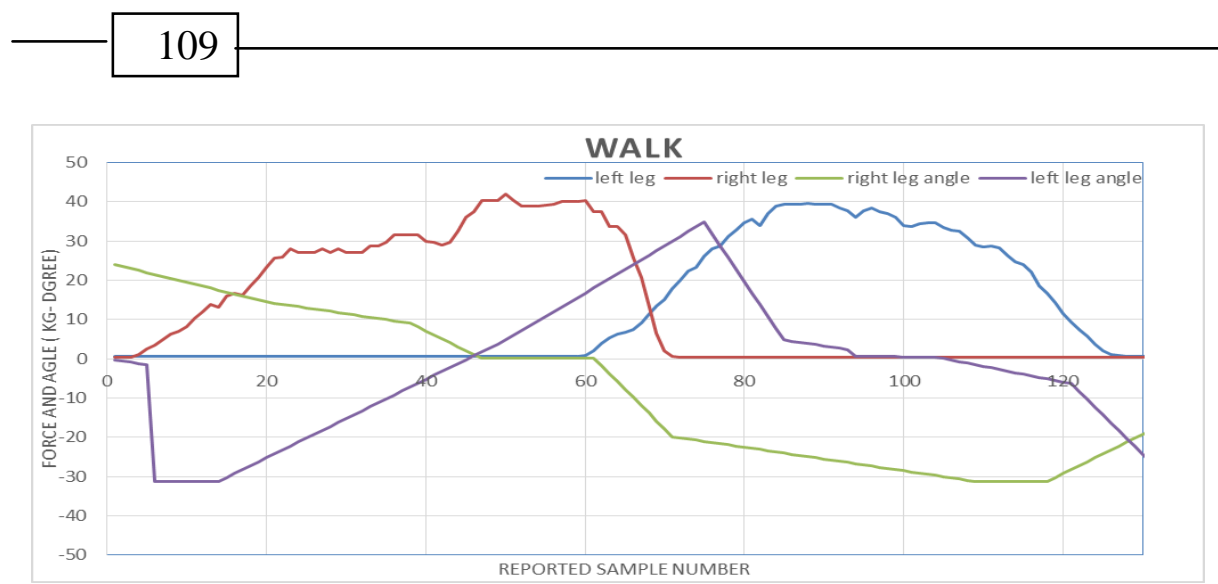

\section{Reference:}

\section{Fig (18)}

1- Abdul Razak, A., Zayegh, A., Begg, R., andWahab, Y.: Foot Plantar Pressure Measurement System: A Review, Sensors, 12, 98849912, https://doi.org/10.3390/s12070 9884, 2012.

2- Amjadi, M., Kyung, K., Park, I., and Sitti, M.: Stretchable, Skin- Mountable, and Wearable Strain Sensors and Their Potential Applications: A Review, Adv. Funct. Mater., 26, 1678-1698, https://doi.org/10.1002/adfm.2 01504755, 2016.

3- Costa, T. and Choi, J.: A flexible two-dimensional force sensor using PDMS nanocomposite, Micro. Eng., 174, 64-69, https://doi.org/10.1016/j.mee.2 017.02.001, 2017.

4- D'Alessio T.: Measurement errors in the scanning of piezoresistive sensors arrays, Sensor. Actuat. A-Phys., 72, 71-76, https://doi.org/10.1016/S09244247(98)00204-0, 1999.

5- Ramalingame, R., Chandraker, P., and Kanoun, O.: Investigation of the influence of solvents in MWCNT-PDMS

nanocomposite pressure sensitive films, Eurosensors 2017 Conference, Paris, France, 3-6 September, Proceedings, MDPI AG., 384, https://doi.org/10.3390/proceed ings 1040384, 2017b.

6- So, H.-M., Sim, J. W., Kwon, J., Yun, J., Baik, S., and Chang, W. S.: Carbon nanotube-based pressure sensor for flexible electronics, Mater. Res. Bull., 48, 5036-5039, https://doi.org/10.1016/j.materr esbull.2013.07.022, 2013. 
7- Someya, T., Kato, Y., Sekitani, T., Iba, D., Noguchi, Y., Murase, Y., Kawaguchi, H., and Sakurai, T.: Conformable, flexible, largearea networks of pressure and thermal sensors with organic transistor active matrixes, P. Natl. Acad. Sci. USA, 102.35, 12321- 12325, https://doi.org/10.1073/pnas.05 02392102, 2005.

8- Wu, J.,Wang, L., Li, J., and Song, A.: A novel crosstalk suppression method of the 2-D networked resistive sensor array, Sensors, 14, 12816-12827, https://doi.org/10.3390/s14071 2816, 2014.

9- Yogeswaran, N., Tinku, S., Khan, S., Lorenzelli, L., Vinciguerra, V., and Dahiya, R.: Stretchable resistive pressure sensor based on CNTPDMS nanocomposites, in 2015 11th Conference on $\mathrm{PhD}$ Research in Microelectronics and Electronics (PRIME), 326- 